\title{
P-055 I - Determinants for insulin secretary capacity and insulin resistance in a rural Japanese area, Okinawa
}

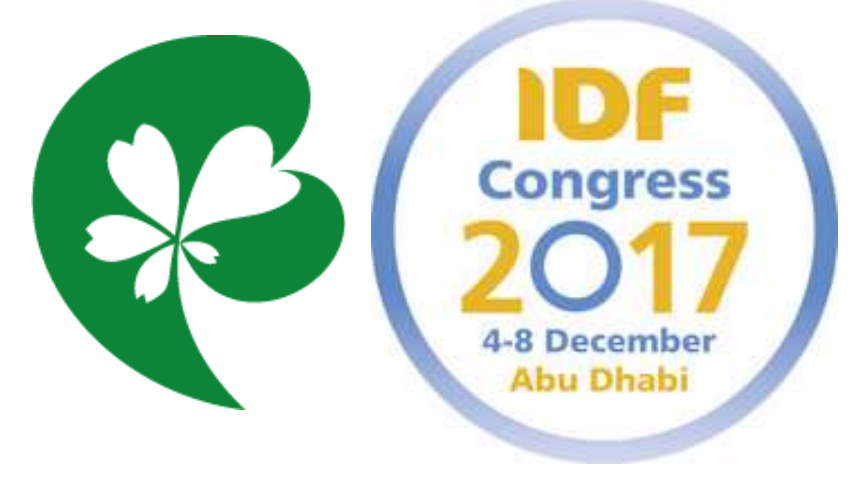

'Diabetes and Lifestyle-related Disease Center, Tomishiro Central Hospital, Okinawa, Japan; ${ }^{2}$ Division of

Endocrinology, Diabetes and Metabolism, Hematology, Rheumatology, Graduate School of Medicine, University of the Ryukyus, Okinawa, Japan; ${ }^{3}$ Department of Diabetes, Endocrinology and Metabolism, Fukushima Medical

University, Fukushima, Japan

\section{Background}

Previous reports on insulin levels after an oral glucose load were controversial; some showed a progressive decline of insulin levels in subjects with normal glucose tolerance, impaired glucose tolerance (IGT) and diabetes, whereas others indicated an increase of insulin levels in IGT and diabetes compared to normal glucose tolerance, during the development from normal glucose tolerance to type 2 diabetes mellitus. Determinants for insulin secretary capacity and insulin resistance might be multifactorial, but precise studies have not been performed.

\section{Purpose}

T To measure determinants for insulin secretary capacity and insulin resistance in a rural Japanese area, Okinawa

\section{Methods}

We continuously recruited $105 \mathrm{I}$ men (mean age 49.2 years) and 895 women (mean age 50.0 years), aged from 30 to 79 years, and compared metabolic parameters, insulin response to an oral glucose load and abdominal fat distribution by CT tomography.

\section{Results}

According to body mass index (BMI: $\left.\mathrm{kg} / \mathrm{m}^{2}\right)$, patients were classified to four groups, $\mathrm{BMI}<25,25 \leqq \mathrm{BMI}<27.5,27.5 \leqq \mathrm{BMI}<30,30 \leqq$ BMI. Visceral fat area (VFA) in each group of subjects was more (I29 vs. 136 vs. 150 vs. 216 ) than estimated by body weight (BW) or body mass index (BMI). Pearson's correlation analysis showed that $\mathrm{I}_{120}$ was positively correlated with $\mathrm{BW}(r=0.348, p=0.047)$, BMI $(r=0.339, p=0.053)$, waist circumference $(W)(r=0.469, p=0.012)$, and VFA $(r=0.619, p=0.01 \mathrm{I})$ in the group of BMls $<25$, however $I_{0}$ and HOMA-IR were not. $\mathrm{I}_{120}$ was not correlated with BW, BMI, W, and VFA in the group of $25 \leqq \mathrm{BMl}<27.5$ and $27.5 \leqq \mathrm{BMI}<30$, but was correlated with BW $(r=0.297, p=0.063)$ and $W(r=0.507$, $\mathrm{P}=0.002)$ in $30 \leqq \mathrm{BMI}$.

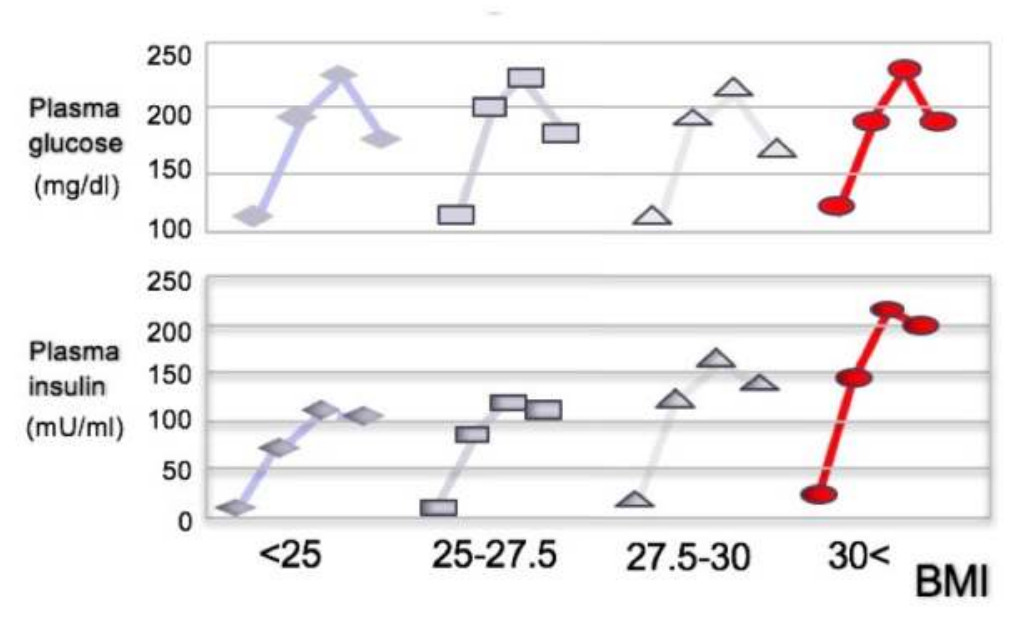

Fig. 1. Responses in plasma glucose and insulin level during $75 \mathrm{~g}$ oral glucose load

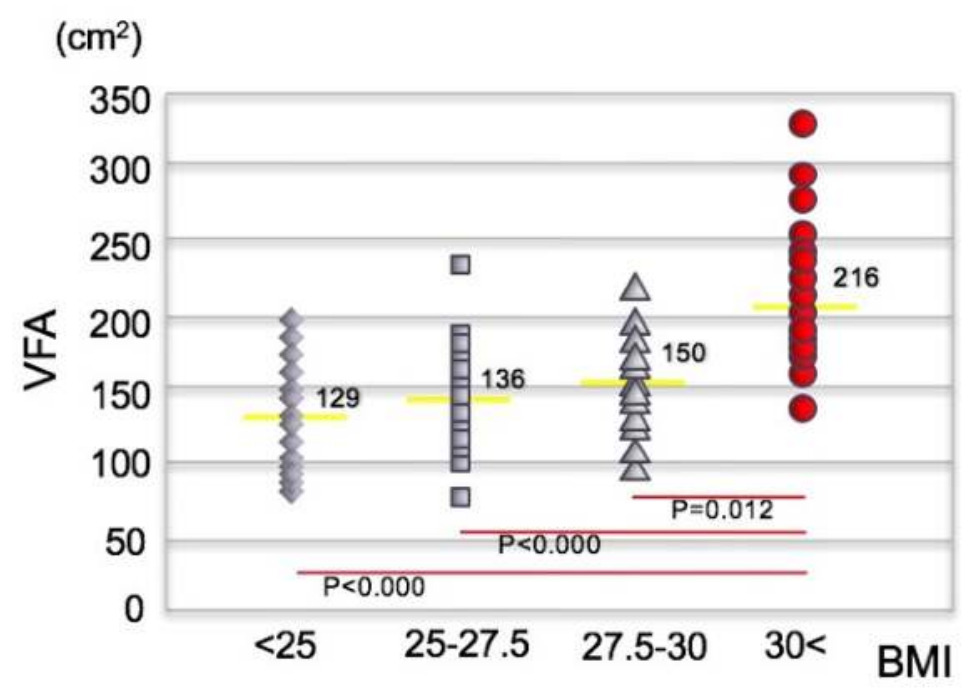

Fig. 2. Visceral fat area was positively correlated with BMI.
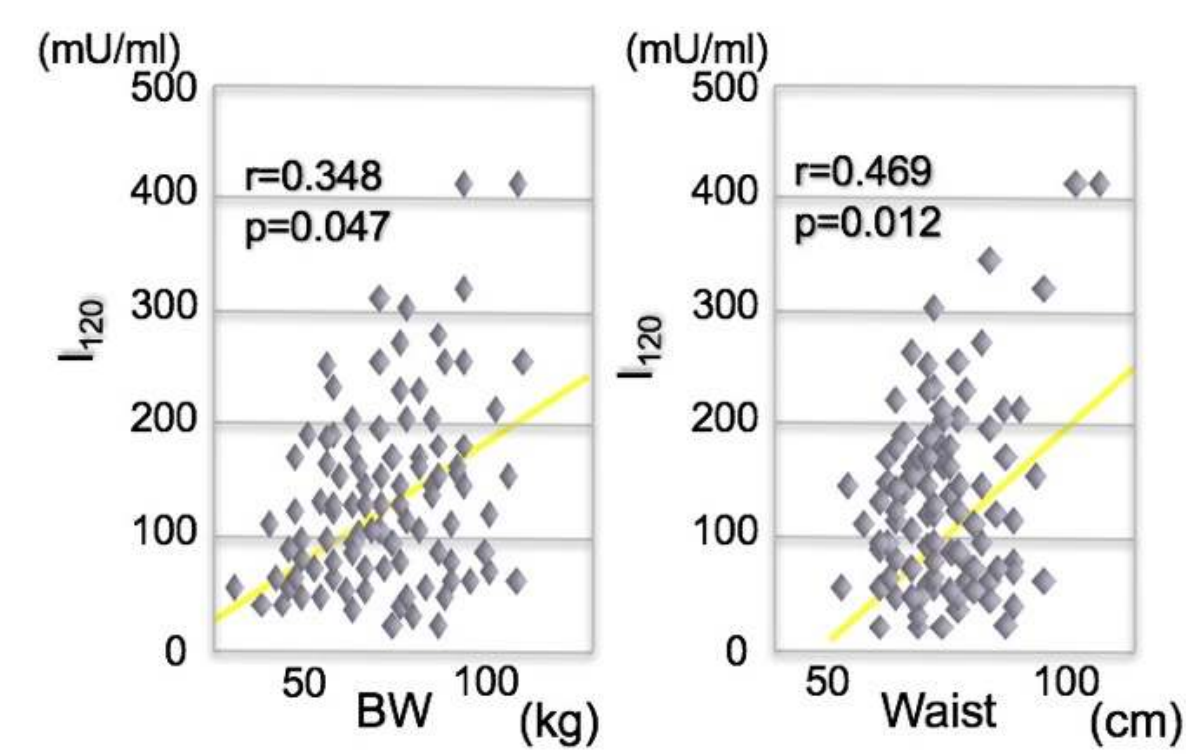

Fig. 3. $I_{120}$ is positively correlated with body weight and waist circumference

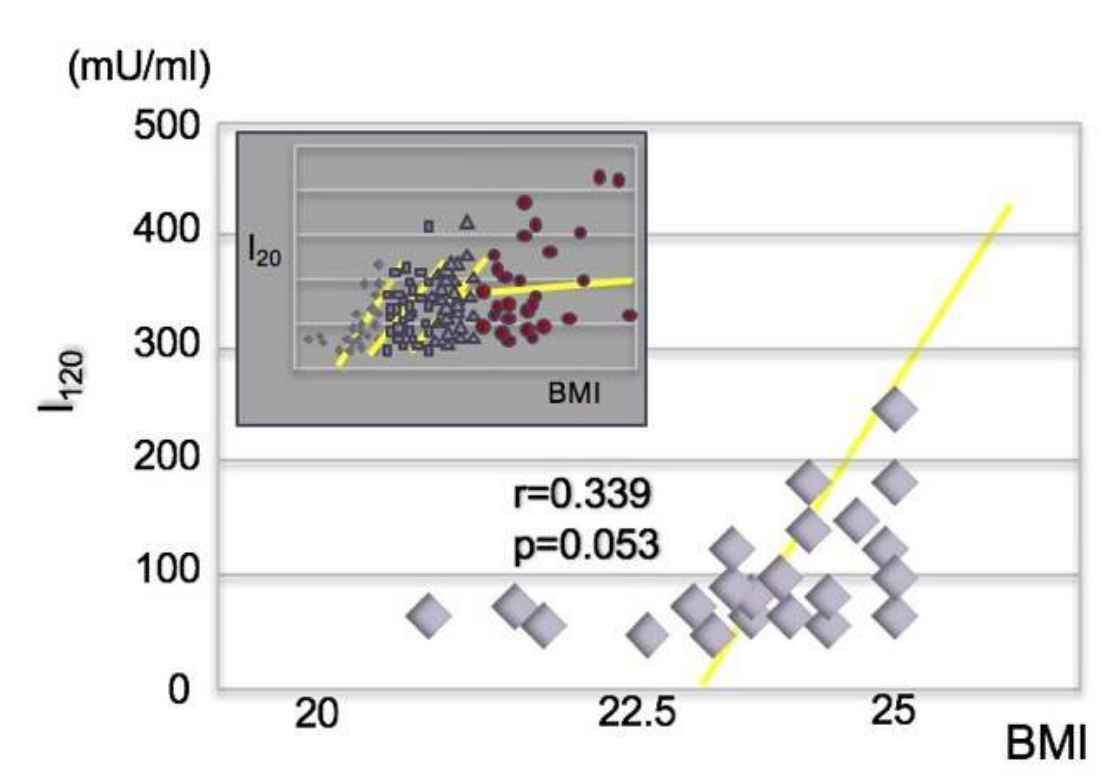

Fig. 4. $I_{120}$ is positively correlated with BMI even in non-obese subjects

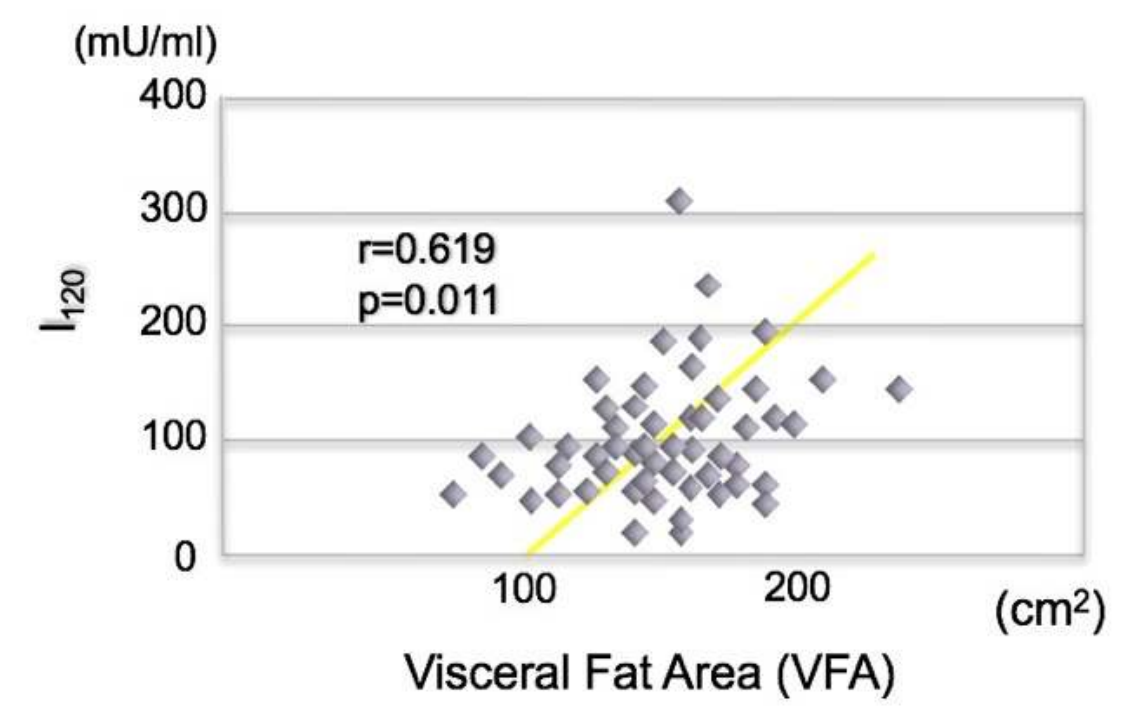

Fig. $5 . I_{120}$ is positively correlated with visceral fat area (VFA)

$\begin{array}{lcc}\mathrm{l}_{120} \text { is positively related with } \\ & \mathrm{r} & \mathrm{p} \\ \text { BMI } & 0.339 & 0.053 \\ \text { Waist } & 0.469 & 0.012 \\ \text { VFA } & 0.619 & 0.011\end{array}$

VFA $>$ Waist $>$ BMI

Fig. 6. Visceral adiposity is associated with delayed hyperinsulinemia in our non-obese subjects.

\section{Conclusion}

We observed that our patients had a higher response of plasma insulin at 2 hours after an oral glucose load $\left(I_{120}\right)$, however Japanese subjects with impaired glucose tolerance was previously reported to be lower insulin response compared to those of the European and American people.Visceral adiposity is associated with delayed hyperinsulinemia in obese subjects but also in non-obese subjects. 